Check for updates

Cite this: New J. Chem., 2019, 43, 1987

Received 6th November 2018 Accepted 23rd December 2018 DOI: $10.1039 / c 8 n j 05640 d$

rsc.li/njc

\section{Mesoporous $\mathrm{ZnMoS}_{4}$ as a supercapacitor electrode material with battery-like behavior}

\author{
Ayman E. Elkholy, (D) a Abdelghaffar S. Dhmees, ${ }^{a}$ Fakiha El-Taib Heakal (D) *b and \\ M. A. Deyab iD a
}

\begin{abstract}
This paper reports the successful synthesis of bimetallic sulfide $Z_{n M o S}$ (ZMS) using a solvothermal method. The structural and morphological properties of ZMS were identified using several characterization methodologies. ZMS material was produced in a sheet-like morphology with a mesoporous structure and its supercapacitive performance was examined. CV and GCD measurements confirmed that ZMS is capable of storing electrical energy showing a battery-like behavior which is expected to proceed via $\mathrm{K}^{+}$ion intercalation/ de-intercalation within the layered matrix of ZMS. Upon raising the current density from 0.7 to $10 \mathrm{~A} \mathrm{~g}^{-1}$, its specific capacitance slightly decreased from $280 \mathrm{~F} \mathrm{~g}^{-1}$ to $243 \mathrm{~F} \mathrm{~g}^{-1}$, maintaining $86.79 \%$ of its initial capacitance, which indicates that the $\mathrm{ZnMoS}_{4}$ compound has a high-rate capability. In addition, the ZMS electrode displays excellent long-term stability preserving a capacitance retention of $\sim 110 \%$ even after 1000 cycles, which is likely attributed to the enhanced diffusion of electrolyte ions within the electrode active material as inferred from EIS measurements.
\end{abstract}

\section{Introduction}

Recently, finding green alternative sources for fossil fuels has become a main concern worldwide. This is because of the catastrophic impacts of excessively emitted greenhouse gases, especially $\mathrm{CO}_{2}$, which are released from the combustion of fossil fuels and contribute significantly to global warming and climatic change. ${ }^{1-4}$ So, today's world needs new approaches for achieving a green and sustainable future with respect to both energy storage and conversion, such as solar energy, fuel cells, water splitting and supercapacitors. ${ }^{5}$ Supercapacitors have unique electrochemical characteristics, such as high specific capacitance, high power density, rapid charge/discharge and a long cycle life. ${ }^{6}$ Therefore, supercapacitors have important applications in the fields of vehicles, electronics and other fields requiring energy storage. ${ }^{7}$ However, the problem of their low energy density has been widely reported, which seriously hinders their commercial, large-scale application. ${ }^{5,7}$ Transitionmetal-based electroactive materials including oxides, nitrides ${ }^{8}$ and sulphides ${ }^{9}$ have drawn great attention as potential electrode materials with high energy density. Transition metal sulfides, particularly those of molybdenum, have triggered intensive interest due to their better electrical conductivity and thermal stability than their corresponding metal oxides and hydroxides. ${ }^{9}$

\footnotetext{
${ }^{a}$ Department of Analysis and Evaluation, Egyptian Petroleum Research Institute, 11727 Cairo, Egypt

${ }^{b}$ Chemistry Department, Faculty of Science, Cairo University, 12613 Giza, Egypt. E-mail: fakihaheakal@yahoo.com
}

Molybdenum sulfide $\left(\mathrm{MoS}_{2}\right)$ is used in many industrial fields including electrochemical devices, hydrogen storage, catalysis and capacitors. It has a framework comparable to graphite, being composed of three atomic layers: a Mo layer sandwiched between two S layers, and the triple layers are linked by weak van der Waals forces. ${ }^{10}$ The current study includes the synthesis of a derivative of $\mathrm{MoS}_{2}$, i.e. bimetallic sulfide $\mathrm{ZnMoS}_{4}$, using a solvothermal method and investigating it as a candidate supercapacitor electrode material. The $\mathrm{Zn}$ metal was selected to be incorporated into the $\mathrm{MoS}_{2}$ matrix, because $\mathrm{Zn}$ ions enhance the electronic conductivity. ${ }^{11}$ The synthesized $\mathrm{ZnMoS}_{4}$ has been characterized for structural and surface morphological properties. Its supercapacitive performance has been examined in $6 \mathrm{M}$ $\mathrm{KOH}$ using cyclic voltammetry (CV), galvanostatic charging/ discharging (GCD) and electrochemical impedance spectroscopy (EIS) techniques.

\section{Materials and experiments}

\subsection{Material synthesis and characterization}

Sodium molybdate $\left(\mathrm{Na}_{2} \mathrm{Mo}_{4} \cdot 6 \mathrm{H}_{2} \mathrm{O}, 99.9 \%\right)$ and zinc acetate dihydrate $\left(\left(\mathrm{CH}_{3} \mathrm{COO}\right)_{2} \mathrm{Zn} \cdot 2 \mathrm{H}_{2} \mathrm{O}, 98.5 \%\right)$ were provided by $\mathrm{BDH}$ Chemical Ltd. Ethanol (99.0\%) and reagent grade ethylene glycol was supplied by Fisher Chemical. Thiourea $\left(\left(\mathrm{NH}_{2}\right)_{2} \mathrm{CS}\right.$, 99\%) was purchased from Merck. All analytical grade chemicals were used as received without further purification. Sodium molybdate with a concentration of $1 \mathrm{mmol}$ and $1 \mathrm{mmol}$ zinc acetate were added together into $40 \mathrm{~mL}$ ethylene glycol solution 
followed by sonication for $30 \mathrm{~min}$ at room temperature. Then, $4 \mathrm{mmol}$ thiourea was added with continuous sonication for another $30 \mathrm{~min}$. The resulting homogenous clear mixture was transferred into a Teflon-lined stainless autoclave (with a $100 \mathrm{~mL}$ capacity), which was kept at $180{ }^{\circ} \mathrm{C}$ for $12 \mathrm{~h}$ in an electric oven. After that, the resultant black powder was collected via centrifugation and washed with water and ethanol. Finally, the product was dried under vacuum conditions at $60{ }^{\circ} \mathrm{C}$ for $12 \mathrm{~h}$.

Several characterization techniques have been adopted for studying the morphology and structural characteristics of the fabricated material. Elemental composition was identified by means of energy-dispersive spectroscopy (EDS) using a Philips XL30 detector (Holland). The material morphology was studied via high-resolution transmission electron microscopy (HR-TEM) using a JEOL JEM-2100 electron microscope (Japan), with an accelerated voltage of $200 \mathrm{keV}$, and the elemental mapping was performed using an Oxford EDS detection unit coupled to this microscope. The crystallographic characteristics of the synthesized material were examined via X-ray diffraction (XRD) using a PANalytical X'pert PRO diffractometer (with $\mathrm{Cu} \mathrm{K} \alpha$ radiation). The $\mathrm{N}_{2}$ gas adsorption/desorption behavior of the prepared material and its specific surface area were investigated using a Quantachrome NOVA Station A (version 11.03).

\subsection{Electrochemical measurements}

All electrochemical measurements performed for $\mathrm{ZnMoS}_{4}$ have been carried out in a $6 \mathrm{M} \mathrm{KOH}$ solution (prepared by dissolving $\mathrm{KOH}$ pellets in deionized water) using a three-electrode cell configuration, including a working electrode, a reference electrode and a counter electrode. The working electrode was prepared using a homogenous slurry of the synthesized ZMS active material (70\%), carbon black (20\%) and poly(vinylidene difluoride) (10\%) in $\mathrm{N}$-methyl-pyrrolidone as a solvent. After continuous stirring for about $48 \mathrm{~h}$ at room temperature, the slurry was spread onto a $\mathrm{Ni}$ foam chip current collector and left to dry at $70^{\circ} \mathrm{C}$. A Pt coil was used as a counter electrode while the reference electrode was an $\mathrm{Ag} / \mathrm{AgCl}$ electrode. The performance of the prepared $\mathrm{ZnMoS}_{4}$ material as a supercapacitor electrode was investigated via cyclic voltammetry (CV), galvanostatic charging/discharging (GCD) and electrochemical impedance spectroscopy (EIS) by means of an electrochemical workstation (IM6e Zahner electrik, Kronach, Germany) driven by Thales software. CV measurements were performed within the potential window of $0.0-0.40 \mathrm{~V}$ at different scan rates $\left(10-100 \mathrm{mV} \mathrm{s}^{-1}\right)$ and the GCD measurements were carried out at different current densities $\left(0.7-10 \mathrm{~A} \mathrm{~g}^{-1}\right)$. The EIS measurements were performed within the frequency range from $10 \mathrm{kHz}$ to $0.1 \mathrm{~Hz}$ at the steady-state open circuit potential with a sinusoidal perturbation amplitude of $10 \mathrm{mV}$. EIS parameters were derived using the EC-Lab V10.40 software.

\section{Results and discussion}

\subsection{Material characterization}

Fig. 1a displays the EDS spectrum of the as-synthesized ZMS material. The peaks equivalent to the elements $\mathrm{Zn}$, Mo and $\mathrm{S}$

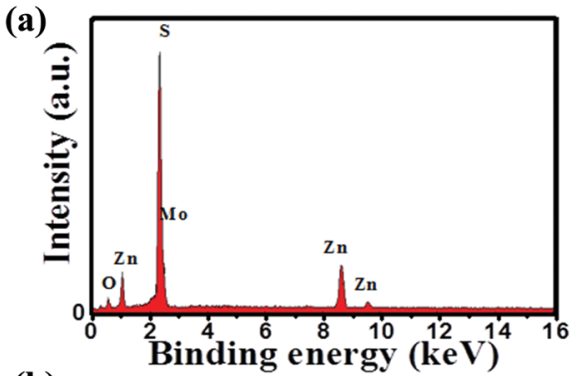

(b)

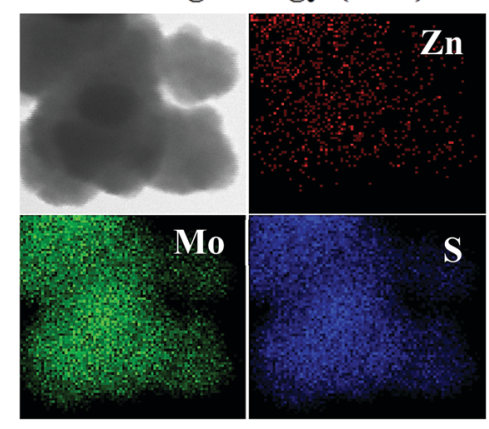

(c)

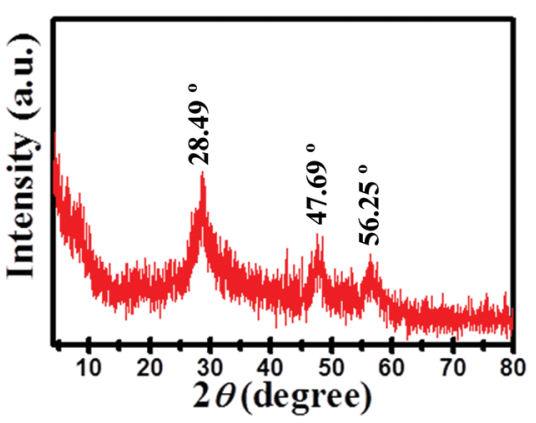

Fig. 1 (a) EDS spectrum, (b) elemental mapping and (c) XRD pattern of the as-synthesized $\mathrm{ZnMoS}_{4}$

indicate their presence in the fabricated material. Fig. 1b shows TEM-EDS elemental mapping which further confirms the uniform distribution of Zn, Mo and S elements within the matrix of this ZMS material. Fig. 1c indicates the XRD pattern of the as-prepared ZMS sample which revealed that there are three main diffraction peaks at $28.49^{\circ}, 47.69^{\circ}$, and $56.25^{\circ}$ matching those of zinc tetrathiomolybdate reported previously in the literature. ${ }^{12,13}$

Fig. 2 shows the HR-TEM images of the as-synthesized ZMS material. The images reveal that our material has been successfully produced in the form of a sheet-like structure. Surface area and pore-size distribution are paramount parameters for energy storage materials, ${ }^{5}$ which can be measured based on the $\mathrm{N}_{2}$ adsorption/ desorption behavior using Brunauer-Emmett-Teller (BET) and Barrett-Joyner-Halenda (BJH) methods, respectively. ${ }^{8}$ Fig. 3a shows the $\mathrm{N}_{2}$ adsorption/desorption isotherm for the fabricated ZMS. This isotherm is of the Type IV characteristic of mesoporous materials. ${ }^{14}$ Moreover, the isotherm is characterized by its hysteresis loop associated with capillary condensation within the mesopores. The initial part shows that adsorption/desorption curves are coincident on each other referring to monolayer-multilayer adsorption. Such features confirm the mesoporous structure of our $\mathrm{ZnMoS}_{4}$ material. Fig. $3 \mathrm{~b}$ shows the pore-size distribution obtained for bimetallic ZMS which reveals an average pore diameter of $3.52 \mathrm{~nm}$, demonstrating 


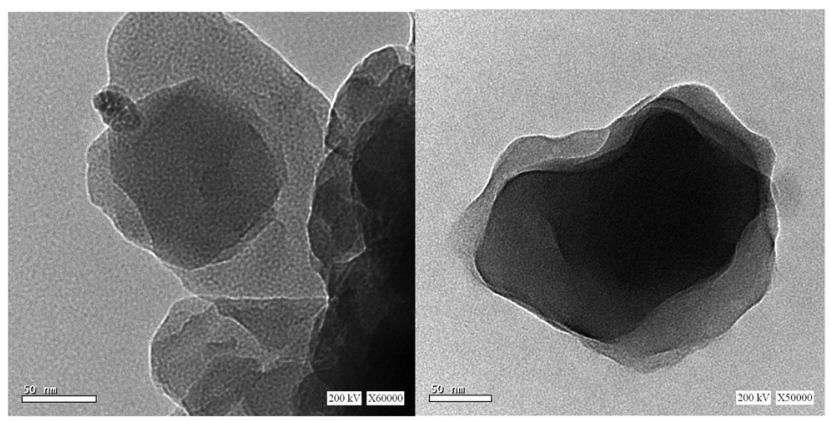

Fig. 2 HR-TEM images of the as-synthesized $\mathrm{ZnMoS}_{4}$.

that the $\mathrm{ZnMoS}_{4}$ material has a mesoporous structure. ${ }^{8}$ As for the BET surface area, it is $49.303 \mathrm{~m}^{2} \mathrm{~g}^{-1}$.

\subsection{Supercapacitive performance}

Many research works in the literature have reported on the supercapacitive performance of metal sulfides, however, most of their results showed a weak performance. Table 1 compiles a comparison between some of these works, including $\mathrm{MoS}_{2}{ }^{15}$ $\mathrm{CuS},{ }^{16} \mathrm{CuSbS}_{2},{ }^{17} \mathrm{WS}_{2},{ }^{18} \mathrm{ZnS}^{19}$ and $\mathrm{Cu}_{2} \mathrm{MoS}_{4} \cdot{ }^{9}$ It is obvious that $\mathrm{ZnMoS}_{4}$ is the most promising material among all listed metal sulfides which may be attributed to the high conductivity provided by Zn. All electrochemical measurements have been undertaken in $6 \mathrm{M} \mathrm{KOH}$ using one compartment three-electrode cell assembly to scrutinize the performance of $\mathrm{ZnMoS}_{4}$ as a potential candidate for a supercapacitor electrode material.

The measurements included cyclic voltammetry (CV), galvanostatic charging discharging (GCD) and electrochemical impedance spectroscopy (EIS), all of which were performed at room temperature $\left(25^{\circ} \mathrm{C}\right)$. $\mathrm{CV}$ is a common technique employed to study the reduction and oxidation processes associated with electroactive species and provides an effective means for categorizing the mode of charge storage. ${ }^{20}$ Electrochemical double layer capacitive materials (carbon-based materials) usually show a rectangular CV shape, while batteries show distinct and widely separated peaks associated with the faradaic oxidation and reduction processes of metal centers involved in the material structure. ${ }^{21}$ Cyclic voltammograms measured for the active $\mathrm{ZnMoS}_{4}$ electrode in $6 \mathrm{M} \mathrm{KOH}$ are displayed in Fig. 4a. There are two distinct redox peaks corresponding to the reduction/oxidation processes
Table 1 Comparison of the specific capacitance of $\mathrm{ZnMoS}_{4}$ and other recently reported metal sulfides

\begin{tabular}{llll}
\hline & Synthetic approach & $\begin{array}{l}\text { Specific } \\
\text { capacitance }\left(\mathrm{F} \mathrm{g}^{-1}\right)\end{array}$ & Ref. \\
\hline $\mathrm{MoS}_{2}$ & Hydrothermal method & 92.85 & 15 \\
$\mathrm{CuS}$ & Sonochemistry & 62.77 & 16 \\
$\mathrm{CuSbS}$ & Colloidal method & 22.00 & 17 \\
$\mathrm{WS}_{2}$ & Chemical exfoliation & 40.00 & 18 \\
$\mathrm{ZnS}_{\mathrm{Cu}_{2} \mathrm{MoS}_{4}}$ & Solvothermal method & 32.80 & 19 \\
$\mathrm{ZnMoS}_{4}$ & Hydrothermal method & 129.68 & 9 \\
& Solvothermal method & 280.00 & Current work
\end{tabular}

occurring for $\mathrm{ZnMoS}_{4}$. Sahoo et al. ${ }^{9}$ have explained the supercapacitive performance of the $\mathrm{Cu}_{2} \mathrm{MoS}_{4}$ material based on the intercalation/de-intercalation of $\mathrm{Na}^{+}$ions diffused from the $\mathrm{Na}_{2} \mathrm{SO}_{4}$ electrolyte within the $\mathrm{Cu}_{2} \mathrm{MoS}_{4}$ electrode. In this regard, the charge storage mechanism of our fabricated $\mathrm{ZnMoS}_{4}$ material can be attributed to the reversible intercalation/de-intercalation of $\mathrm{K}^{+}$ions obtained from the $\mathrm{KOH}$ electrolyte occurring at the ZMS electrode according to the following equation:

$$
\mathrm{ZnMoS}_{4}+\mathrm{K}^{+}+\mathrm{e} \leftrightarrow \mathrm{KZnMoS}_{4}
$$

In this mechanism, $\mathrm{K}^{+}$ions get intercalated within $\mathrm{ZnMoS}_{4}$ sheets during the charging step, which mainly relies on the redox system $\mathrm{Mo}(\mathrm{vI}) / \mathrm{Mo}(\mathrm{v})\left(\mathrm{Mo}^{6+}+\mathrm{e} \leftrightarrow \mathrm{Mo}^{5+}\right)$, because the Mo element can be interchangeable through several oxidation states, and the case is reversed during the discharging step. So, the obtained redox peaks may be attributable to subsequent oxidation/reduction of the central Mo metal in the sulfide structure. The obtained redox peaks are broad and distinct indicating a battery-like behavior associated with faradaic-like features. ${ }^{8}$ The CV curves have been recorded as a function of the scan rate $(v)$ within the range of 10 to $100 \mathrm{mV} \mathrm{s}^{-1}$, where it was found that peak-to-peak separation $\left(\Delta E_{\mathrm{p}-\mathrm{p}}\right)$ increases with increasing scan rate. For example, when the scan rate increases from 10 to $100 \mathrm{mV} \mathrm{s}^{-1}$, the anodic peak potential $\left(E_{\mathrm{p}, \mathrm{a}}\right)$ is changed from $0.299 \mathrm{~V}$ to $0.330 \mathrm{~V}$ ( $v s$. Ag/AgCl). Such an effect results from the insufficient access of ions from the electrolyte to the electrode surface at high scan rates and increased internal diffusion resistance, ${ }^{22}$ while $I_{\mathrm{p}}$ increases with $v$ due to the fast interfacial kinetics. ${ }^{23}$ It is worth mentioning that the similarity of both the anodic and cathodic profiles refers to the
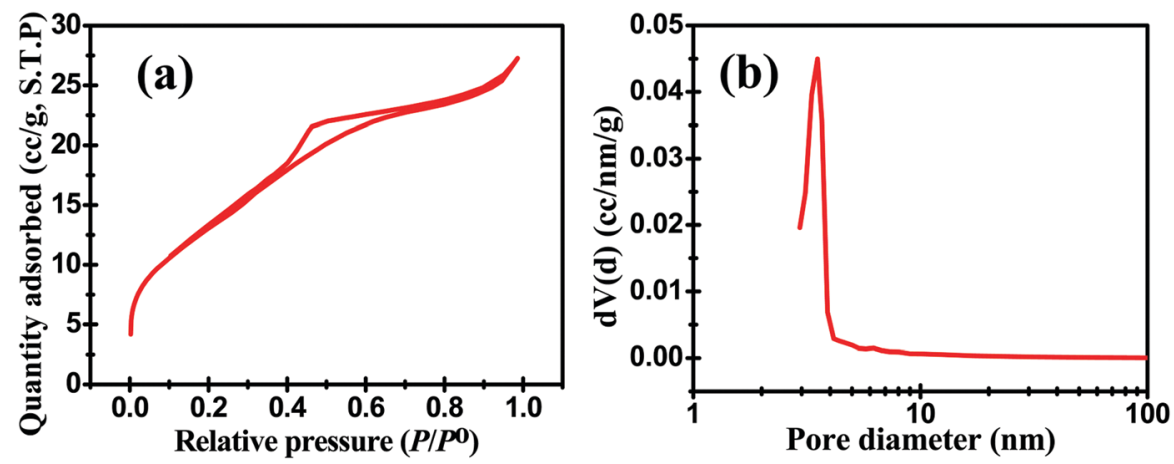

Fig. 3 (a) $\mathrm{N}_{2}$ adsorption/desorption isotherm and (b) BJH pore size distribution for the as-synthesized $\mathrm{ZnMoS}_{4}$. 
(a)

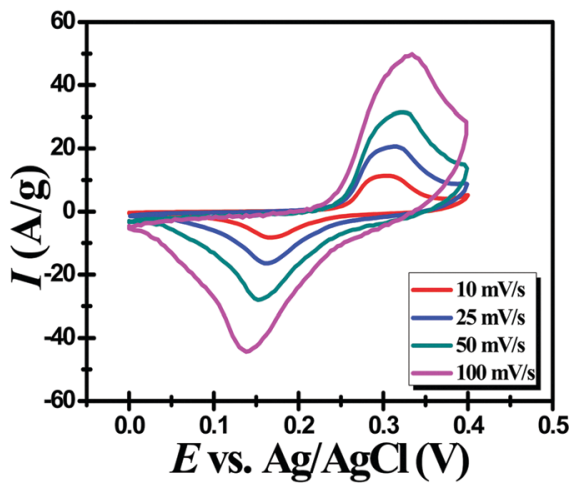

(b)

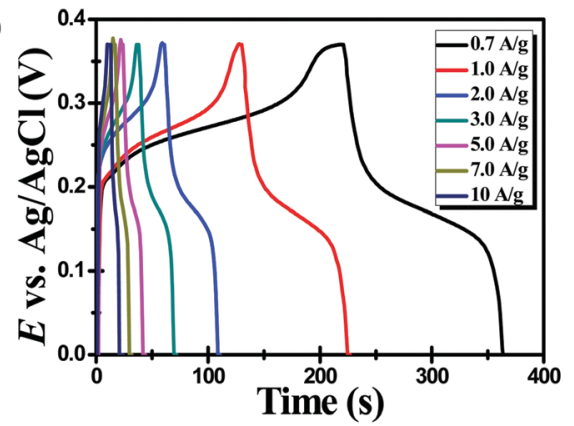

(c)

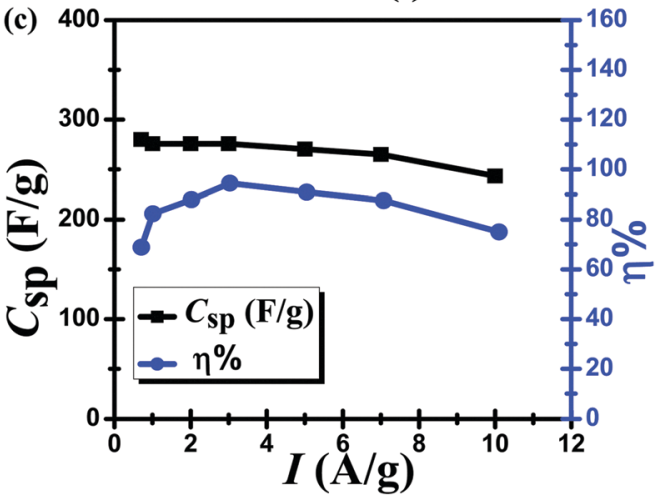

Fig. 4 Electrochemical characterization of the $\mathrm{ZnMoS}_{4}$ electrode in $6 \mathrm{M} \mathrm{KOH}$ electrolyte: (a) CV curves as a function of scan rate, (b) GCD curves as a function of current density and (c) the calculated $C_{\mathrm{sp}}$ and $\eta \%$ at different current densities.

reversibility of the redox reactions associated with our ZMS active material. $^{8}$

In order to investigate the storage capability of the ZMS material for electrical energy, galvanostatic charge/discharge (GCD) measurement was performed to calculate the specific capacitance associated with it. The GCD technique is an accurate method often utilized to determine the electrochemical supercapacitive performance of electroactive materials. ${ }^{24}$ The GCD output measurement is the chronopotentiometry ( $E v s$. time) relationship, where a constant specific current is applied on the material and the potential response is recorded with time. For carbonaceous materials, constant-current charging/discharging results in a linear ( $E$ vs. $t$ ) plot for each anodic/cathodic curve (i.e. a triangular-shaped GCD curve). It is unlikely that the GCD curve for batteries becomes nonlinear which is characterized by the presence of plateaus of nearly constant potentials corresponding to the potentials at which the faradaic oxidation/reduction of the metal centers occurs $^{21}$ as shown in Fig. 4b which displays the GCD curves recorded for the ZMS material measured at different current densities from $0.7 \mathrm{~A} \mathrm{~g}^{-1}$ to $10 \mathrm{~A} \mathrm{~g}^{-1}$, which also indicates the features of battery-like behavior. ${ }^{25}$ The ZMS electrode consumes longer times for both charging and discharging at lower current densities, which is attributed to the enhanced electrolyte/electrode interactions at low current densities. The specific capacitance is calculated at each current density using the discharging times obtained from GCD curves according to eqn (2): ${ }^{23,26,27}$

$$
C_{\mathrm{sp}}=\frac{I \Delta t}{m \Delta V}
$$

where $I$ is the applied current (A), $m$ is the mass of active material (g) and $\Delta V$ is the potential window $(\mathrm{V})$. Fig. $4 \mathrm{c}$ shows the dependence of the calculated specific capacitance on the applied current density. Surprisingly, although the current density has been changed from $0.7 \mathrm{~A} \mathrm{~g}^{-1}$ to $10 \mathrm{~A} \mathrm{~g}^{-1}$, the specific capacitance slightly decreased from $280 \mathrm{~F} \mathrm{~g}^{-1}$ to $243 \mathrm{~F} \mathrm{~g}^{-1}$, i.e. this material is capable of maintaining $86.79 \%$ of its initial capacitance. Such behavior indicates that the as-synthesized ZMS has an effective storage capacity with excellent rate capability. ${ }^{28}$ In order to calculate the coulombic efficiency ( $\eta \%)$ of the charging/discharging process for ZMS, eqn (3) was applied using the charging and discharging times obtained from GCD measurements as follows: ${ }^{24,29}$

$$
\eta \%=\frac{Q_{\mathrm{d}}}{Q_{\mathrm{c}}} \times 100 \rightarrow \eta \%=\frac{t_{\mathrm{d}}}{t_{\mathrm{c}}} \times 100
$$

where $Q=I t$ and $I_{\mathrm{c}}=I_{\mathrm{d}}, Q_{\mathrm{c}}$ and $Q_{\mathrm{d}}$ are, respectively, the amount of electrical charges stored in or released from the material, and $t_{\mathrm{c}}$ and $t_{\mathrm{d}}$ are the times of charging and discharging obtained at the same current density, respectively. $\eta \%$ has its maximum value at intermediate current densities reaching $94.44 \%$ at $3 \mathrm{~A} \mathrm{~g}^{-1}$, while it decreases at lower and higher current densities becoming $75 \%$ and $68.84 \%$ at $10 \mathrm{~A} \mathrm{~g}^{-1}$ and $0.7 \mathrm{~A} \mathrm{~g}^{-1}$, respectively.

It is a paramount requirement to test the cycling stability and durability of supercapacitor electrode materials to ensure their good performance, especially during long-term use. This test has been performed using cyclic voltammetry, ${ }^{30}$ where the ZMS electrode was subjected to $1000 \mathrm{CV}$ cycles at a scan rate of $50 \mathrm{mV} \mathrm{s}^{-1}$. Fig. 5a shows 5 selected CV cycles (the 3rd, 50th, 100th, 500th and 1000th cycles) from the 1000 cycles applied on the ZMS electrode. Obviously, the area under the CV curves is increased by increasing the number of voltammograms. The specific capacitance was calculated from the applied CV measurement according to eqn (4). ${ }^{31,32}$

$$
C_{\mathrm{sp}}=\frac{\int I(\mathrm{~d} V)}{m v \Delta V}
$$

where $I$ is the response current (A), $m$ is the mass of the active material $(\mathrm{g}), v$ is the scan rate $\left(\mathrm{mV} \mathrm{s}^{-1}\right)$ and $\Delta V$ is the potential window (V). Amazingly, the calculated specific capacitance (Fig. 5b) was found to be continuously rising as long as the $\mathrm{CV}$ measurement proceeds. For example, the initially calculated $C_{\mathrm{sp}}$ is $257 \mathrm{~F} \mathrm{~g}^{-1}$, but it rises to $282 \mathrm{~F} \mathrm{~g}^{-1}$ after $1000 \mathrm{CV}$ cycles. Such behavior can be explained on the basis that the ZMS material is continually activated during the course of charging/discharging due to successive oxidation/reduction processes that enhance the 

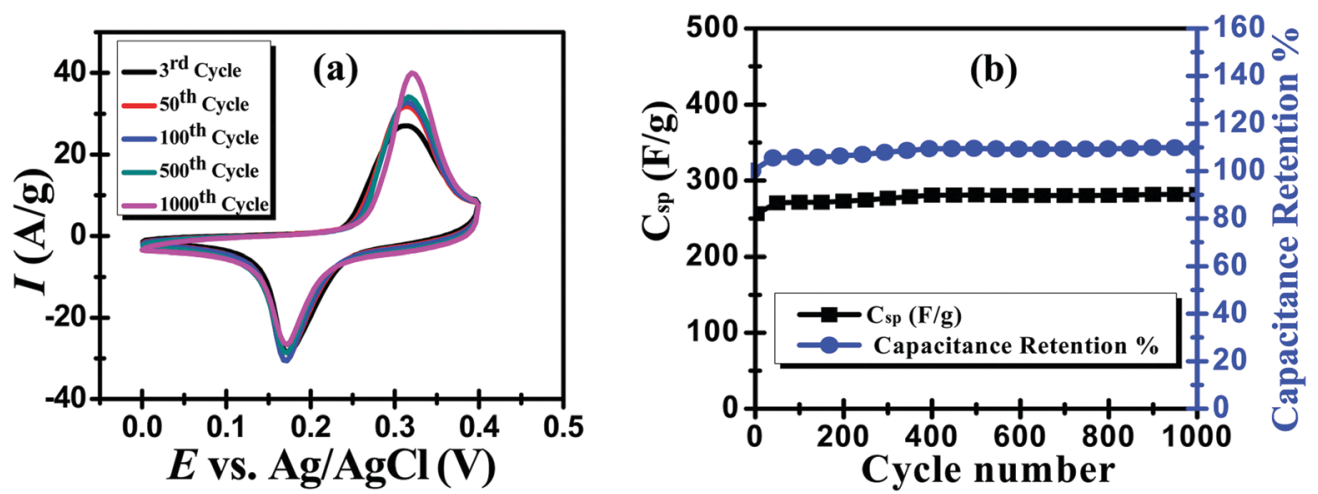

Fig. 5 (a) Cycling stability test using $\mathrm{CV}$ measurements for 1000 cycles at a scan rate of $50 \mathrm{mV} \mathrm{s}^{-1}$ and (b) the correspondingly calculated $C_{\mathrm{sp}}$ and capacitance retention.

electrode/electrolyte interactions. Accordingly, the capacitance retention was found to rise continually, reaching $\sim 110 \%$ after $1000 \mathrm{CV}$ cycles, and this behavior is likely to be attributed to the electrochemical activation of the ZMS electrode. ${ }^{5}$ Electrode activation may have occurred due to the cleaning of its surface by the action of successive charging/discharging, and this assumption is supported by the enhanced electrolyte diffusion within the ZMS matrix as inferred from EIS measurements as will be explained below.

Electrochemical impedance spectroscopy (EIS) was executed to deeply investigate the electrochemical performance of ZMS during long-term use. EIS is a useful technique that can provide valuable information for understanding electrode/electrolyte interactions. ${ }^{22}$ Fig. 6a shows the EIS spectra of the ZMS electrode in the form of Nyquist plots before and after the cycling stability test within the frequency range from $10000 \mathrm{~Hz}$ to $0.1 \mathrm{~Hz}$. Each spectrum indicates a semi-straight line, but on zooming in on the high-frequency region $(>200 \mathrm{~Hz})$, one can note that there is a small part of a semi-circuit for each curve as shown in the inset of Fig. 6a. So, it is crucial to undertake a fitting process so as to obtain tangible outputs (numerical values) to easily describe the electrochemical behavior of the ZMS material. To achieve that, an equivalent electrical circuit was suggested (as depicted in Fig. 6b) to simulate the electrochemical process across the electrode/ electrolyte interface. This model comprises a resistor $\left(R_{1}\right)$ in series with a time constant $\left(Q /\left(R_{2}+W\right)\right)$.
Fig. 6b also displays the regions within the $\mathrm{ZnMoS}_{4}$ electrode equivalent to each part of the suggested equivalent circuit, where $R_{1}$ represents the bulk resistance of the $\mathrm{KOH}$ electrolyte and $\left(Q /\left(R_{2}+W\right)\right)$ is a time constant equivalent to the $\mathrm{KOH} /$ $\mathrm{ZnMoS}_{4}$ interface and the $\mathrm{ZnMoS}_{4}$ electrode bulk. Certainly, the resistance of the current collector (the $\mathrm{Ni}$ foam) is negligible as the resistance of metals is considered to be equal to zero in general. The element representing Warburg impedance was included in this model due to the obtained semi-straight part in the EIS spectra in the lowest-frequency region, which indicates that the electrochemical process associating $\mathrm{ZnMoS}_{4}$ is diffusion-controlled. ${ }^{10}$ Table 2 lists the fitting outputs of EIS spectra before and after undertaking the cycling stability test. The solution resistance $\left(R_{1}\right)$ has suffered a very minute change (from $0.24 \Omega$ to $0.29 \Omega$ ). The charge transfer resistance $\left(R_{2}\right)$, which describes the accumulation of electrolyte ions across the $\mathrm{KOH} / \mathrm{ZnMoS}_{4}$ interface, increased from $0.49 \Omega$ to $35.60 \Omega$. The total resistance (which is a summation of $R_{1}$ and $R_{2}$ ) represents the equivalent series resistance (ESR), which is an important parameter for generally evaluating storage materials. ${ }^{33}$ Herein, the ESR of the ZMS electrode has suffered an insignificant increase (from $0.73 \Omega$ to only $35.89 \Omega$ ), which further confirms the outperformance of the ZMS material. More interestingly, the value of $W$, representing the hindrance of the ZMS material to electrolyte diffusion, has significantly dropped from $475.70 \Omega \mathrm{s}^{-0.5}$

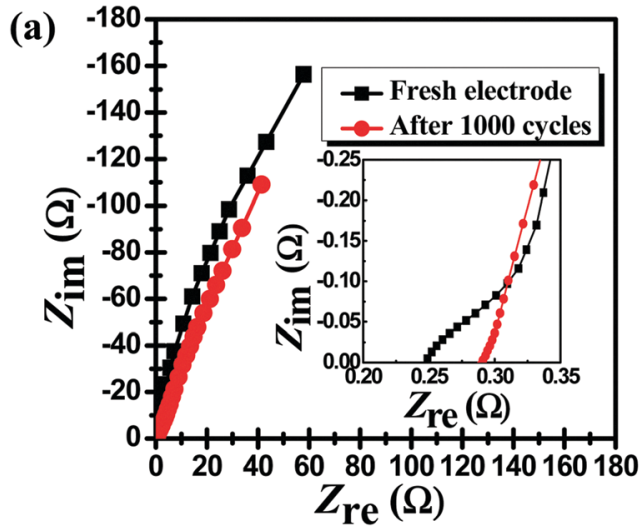

(b)

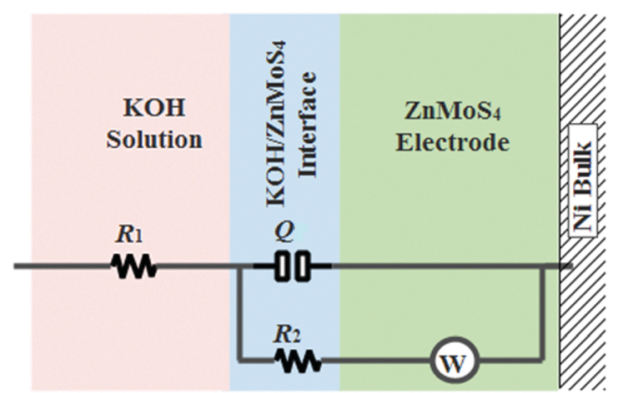

Fig. 6 (a) Nyquist spectra recorded before and after 1000 cycles and (b) the equivalent circuit modeling the EIS spectra. 
Table 2 EIS parameters calculated for the $\mathrm{ZnMoS}_{4}$ electrode before and after $1000 \mathrm{CV}$ cycles

\begin{tabular}{lll}
\hline & Fresh electrode & After 1000 cycles \\
\hline$R_{1}(\Omega)$ & 0.24 & 0.29 \\
$Q(\mathrm{mF})$ & 8.07 & 10.61 \\
$n$ & 0.92 & 0.85 \\
$R_{2}(\Omega)$ & 0.49 & 35.60 \\
$W\left(\Omega \mathrm{s}^{-0.5}\right)$ & 475.70 & 293.30 \\
\hline
\end{tabular}

to $293.30 \Omega \mathrm{s}^{-0.5}$, which may explain why the specific capacitance has increased after long-term use, i.e. due to the enhanced electrolyte diffusion within the electrode material.

\section{Conclusions}

In this work, the bimetallic sulfide $\mathrm{ZnMoS}_{4}$ material was successfully synthesized in a sheet-like structure using a solvothermal method and assessed as a candidate for supercapacitor applications in $6 \mathrm{M} \mathrm{KOH}$ using $\mathrm{CV}$, GCD and EIS measurements in a one compartment three-electrode cell configuration. Cyclic voltammograms displayed broad and distinct peaks attributed to the oxidation-reduction processes associated with the redox system $\mathrm{Mo}(\mathrm{vI}) / \mathrm{Mo}(\mathrm{v})$ in the fabricated ZMS material, indicating a battery-like behavior. GCD results confirmed such a behavior with a calculated specific capacitance of $280 \mathrm{~F} \mathrm{~g}^{-1}$ at a current density of $0.7 \mathrm{~A} \mathrm{~g}^{-1}$. A long-term CV-based test showed excellent cycling stability with a capacitance retention reaching $\sim 110 \%$ even after 1000 cycles. EIS measurements revealed that electrolyte diffusion was improved during the long-term cycling which could explain the excellent cycling stability of the ZMS electrode.

\section{Conflicts of interest}

There are no conflicts to declare.

\section{Acknowledgements}

The authors gratefully acknowledge the research support provided by the Faculty of Science, Cairo University and the Egyptian Petroleum Research Institute.

\section{References}

1 S. Wang, T. Wang, P. Liu, Y. Shi, G. Liu and J. Li, Mater. Res. Bull., 2017, 88, 62-68.

2 C. Khan, R. Amin and G. Madden, Egypt. J. Pet., 2013, 22, 225-240.

3 A. Raza, R. Rezaee, C. H. Bing, R. Gholami, M. A. Hamid and R. Nagarajan, Egypt. J. Pet., 2016, 25, 367-373.

4 A. M. Elfadly, A. M. Badawi, F. Z. Yehia, Y. A. Mohamed, M. A. Betiha and A. M. Rabie, Egypt. J. Pet., 2013, 22, 373-380.

5 Y. Shabangoli, M. S. Rahmanifar, M. F. El-Kady, A. Noori, M. F. Mousavi and R. B. Kaner, Energy Storage Mater., 2018, 11, 282-293.
6 M. Lu, X.-P. Yuan, X.-H. Guan and G.-S. Wang, J. Mater. Chem. A, 2017, 5, 3621-3627.

7 A. Yu, V. Chabot and J. Zhang, Electrochemical supercapacitors for energy storage and delivery: fundamentals and applications, CRC Press, New York, 2013.

8 A. E. Elkholy, F. El-Taib Heakal and N. K. Allam, RSC Adv., 2017, 7, 51888-51895.

9 S. Sahoo, K. Krishnamoorthy, P. Pazhamalai, V. K. Mariappan and S. J. Kim, Int. J. Hydrogen Energy, 2018, 43, 12222-12232.

10 K.-J. Huang, L. Wang, Y.-J. Liu, Y.-M. Liu, H.-B. Wang, T. Gan and L.-L. Wang, Int. J. Hydrogen Energy, 2013, 38, 14027-14034.

11 S. G. Mohamed, S. Y. Attia and N. K. Allam, Mater. Today Energy, 2017, 4, 97-104.

12 G. H. Guo, Y. T. Tao, Z. P. Song and K. L. Zhang, J. Solid State Electrochem., 2005, 11, 90-92.

13 V. S. Perera, N. P. Wickramaratne, M. Jaroniec and S. D. Huang, J. Mater. Chem. B, 2014, 2, 257-261.

14 K. S. Sing, Pure Appl. Chem., 1985, 57, 603-619.

15 K. Krishnamoorthy, G. K. Veerasubramani, S. Radhakrishnan and S. J. Kim, Mater. Res. Bull., 2014, 50, 499-502.

16 K. Krishnamoorthy, G. K. Veerasubramani, S. Radhakrishnan and S. J. Kim, J. Nanosci. Nanotechnol, 2015, 15, 4409-4413.

17 K. Ramasamy, R. K. Gupta, S. Palchoudhury, S. Ivanov and A. Gupta, Chem. Mater., 2014, 27, 379-386.

18 C. C. Mayorga-Martinez, A. Ambrosi, A. Y. S. Eng, Z. Sofer and M. Pumera, Electrochem. Commun., 2015, 56, 24-28.

19 R. Ramachandran, M. Saranya, P. Kollu, B. P. C. Raghupathy, S. K. Jeong and A. N. Grace, Electrochim. Acta, 2015, 178, 647-657.

20 N. m. Elgrishi, K. J. Rountree, B. D. McCarthy, E. S. Rountree, T. T. Eisenhart and J. L. Dempsey, J. Chem. Educ., 2017, 95, 197-206.

21 Y. Gogotsi and R. M. Penner, ACS Nano, 2018, 12, 2081-2083.

22 A. E. Elkholy, F. El-Taib Heakal and N. K. Allam, Electrochim. Acta, 2019, 296, 59-68.

23 L. Liu, H. Zhang, Y. Mu, Y. Bai and Y. Wang, J. Power Sources, 2016, 327, 599-609.

24 W. Wang, Q. Hao, W. Lei, X. Xia and X. Wang, J. Power Sources, 2014, 269, 250-259.

25 C. Liu and X. Wu, Mater. Res. Bull., 2018, 103, 55-62.

26 Z. Wang, W. Jia, M. Jiang, C. Chen and Y. Li, Nano Res., 2016, 9, 2026-2033.

27 M. M. Vadiyar, S. S. Kolekar, J.-Y. Chang, A. A. Kashale and A. V. Ghule, Electrochim. Acta, 2016, 222, 1604-1615.

28 F. M. Ismail, M. Ramadan, A. M. Abdellah, I. Ismail and N. K. Allam, J. Electroanal. Chem., 2018, 817, 111-117.

29 A. Rai, A. L. Sharma and A. K. Thakur, Solid State Ionics, 2014, 262, 230-233.

30 M. L. Aparna, A. N. Grace, P. Sathyanarayanan and N. K. Sahu, J. Alloys Compd., 2018, 745, 385-395.

31 K. Bimdu, K. Sridharan, K. M. Ajith, H. N. Lim and H. S. Nagaraja, Electrochim. Acta, 2016, 217, 139-149.

32 P. Xiong, H. Huang and X. Wang, J. Power Sources, 2014, 245, 937-946.

33 P. Guo, Z. Li, S. Liu, J. Xue, G. Wu, H. Li and X. S. Zhao, J. Mater. Sci., 2017, 52, 5359-5365. 